\title{
Modeling the Air-Ground Multipath Channel
}

\author{
Nicolas Schneckenburger ${ }^{1}$, Thomas Jost ${ }^{1}$, Uwe-Carsten Fiebig ${ }^{1}$, \\ Giovanni Del Galdo ${ }^{2,3}$, Hosseinali Jamal ${ }^{4}$, David Matolak ${ }^{4}$, Ruoyu Sun ${ }^{5}$ \\ ${ }^{1}$ Institute of Communications and Navigation, Deutsches Zentrum für Luft- und Raumfahrt (DLR), Weßling, Germany \\ ${ }^{2}$ Institute for Information Technology, Ilmenau University of Technology, Ilmenau, Germany \\ ${ }^{3}$ Fraunhofer Institute for Integrated Circuits IIS, Erlangen, Germany \\ ${ }^{4}$ University of South Carolina, Columbia, SC, USA \\ ${ }^{5}$ National Institute of Standards and Technology (NIST), Boulder, CO, USA
}

\begin{abstract}
The paper proposes a geometrical-statistical modeling approach for the air-ground channel for communications, navigation, and surveillance (CNS) systems in the L-band frequency range. We sketch the architecture of the model with its six elements and show how their parameters can be derived from measurement data. Preliminary results obtained from a relatively small set of measurement data reveal that the proposed modeling approach is well suited to capture the time variant behavior of the channel. However, a considerably more extensive evaluation of the measurement data will be necessary to finalize the parameter settings of the proposed channel model.
\end{abstract}

\section{INTRODUCTION}

The communications, navigation, and surveillance (CNS) infrastructure in civil aviation is currently undergoing a major innovation process to allow higher traffic levels and more efficient flight operations.

On the communication side, new ground based systems are being developed to replace the analog very high frequency (VHF) voice link [1]. As for navigation, alternative positioning navigation and timing (APNT) systems are used as backup in case the primary satellite based navigation infrastructure becomes unavailable [2], [3]. All these ground based communication and navigation systems are assigned to use the L-band frequency range and usually have bandwidths below $1 \mathrm{MHz}$. In order to guarantee reliable communications between ground and air and allow for accurate positioning, it is crucial to understand and model the propagation characteristics of the air-ground (AG) radio channel. Hereby, multipath propagation plays a major role, as it can degrade both communication and navigation performance [4], [5].

A general and accurate model of the AG channel is mandatory for the testing and optimization of both modified legacy as well as novel CNS systems. An AG channel model allows the analysis of terrestrial CNS independently from flight trials which are usually very complex, cost intensive, and require a long lead time. Additionally, the number of flight trajectories, systems or configurations which can be covered by flight trials is limited. Using a channel model, terrestrial systems can be evaluated using computer simulations. Simulations allow the economic and rapid testing of a large variety of systems and configurations. Currently, no accurate general wideband model of AG channel derived from measurement data exists for analyzing CNS system performance.
In this paper, we present the architecture of a new model for the AG channel. The modeling is based on $10 \mathrm{MHz}$ channel sounding flight measurements conducted in the L-band. The main benefit of the presented AG channel modeling approach is its flexibility to be adapted to a large range of different environments a ground station can be setup in. Thanks to a geometrical description, the AG channel model is better suited to describe not only the statistical channel properties but also the time evolution of the various propagation effects. Additionally, compared to a purely statistical approach, a geometry-based stochastic channel model (GSCM) models propagation effects closer to their original physical cause; e.g. a multipath component (MPC) is not modeled as a purely statistical event but as a reflection originating from a specific building. The modeling approach is a profound basis for the performance evaluation of terrestrial CNS systems.

The paper is organized as follows: Sec. II provides an overview of the model architecture, describes how the different propagation effects can be modeled, and outlines how the model parameters can be derived from measurement data. In Sec. III we give preliminary results on the propagation conditions resulting from the presented modeling approach. The paper concludes with a brief discussion and outlines future work in Sec. IV.

\section{Archichtecture of the Channel Model}

In the following section we give an overview of the GSCM architecture. The overview includes a description of the different modeling elements and outlines how their parameters can be derived from measurement data.

The model architecture is designed with a geometrical statistical approach: the dominant propagation effects are modeled deterministically by means of simple geometrical elements whose parameters, e.g., the position of a reflector, are randomly drawn from statistical distributions. These distributions are derived by the analysis of measurement data. Different sets of distributions allow us to use the very same architecture to model very different scenarios. The modeling framework is very versatile as it includes all relevant propagation effects which have been observed during measurements [6], [7] covering a wide range of environments.

Fig. 1 shows the proposed architecture of the channel model. The general idea is to model the propagation channel as a sum 


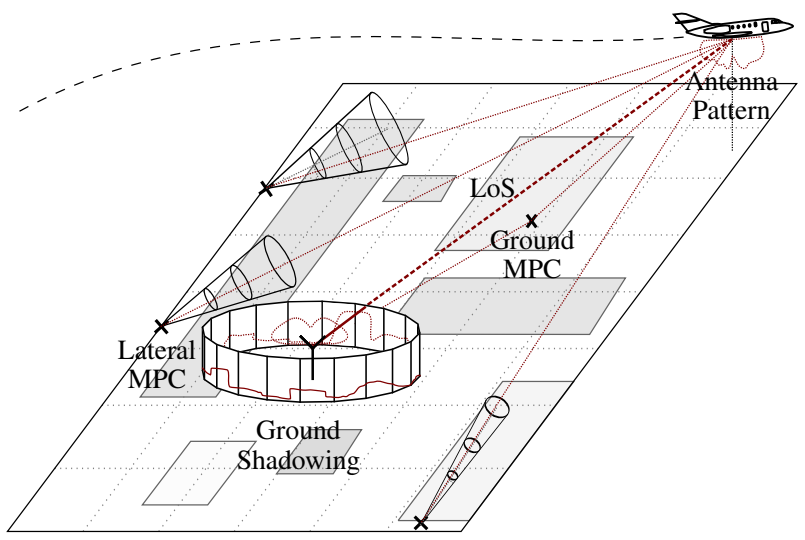

Fig. 1: Proposed GSCM approach for the AG channel.

of plane waves. The waves can originate from different effects, such as line-of-sight (LoS) propagation, ground multipath propagation, and lateral multipath propagation, described in sections Sec. II-A through Sec. II-C. Moreover, propagation paths are weighted by an antenna pattern at the aircraft and ground station described in Sec. II-D. Additionally, the LoS path may be blocked by the effect of ground shadowing as outlined in Sec. II-E. The colored noise component described in Sec. II-F models non specular or dense MPCs.

\section{A. Line-of-sight (LoS) Path}

The LoS path is the direct propagation path between a ground station and aircraft. Under normal conditions the majority of power is received through the LoS signal. The received power of the LoS signal follows free space path loss (FSPL) conditions (if not influenced by any of the effects described below). For navigation applications, the bending of the electromagnetic waves due to the tropospheres composition leads to a slight overestimation of the range. The increasing speed of light for higher layers of the troposphere has the opposite effect, as it cause ranges to be estimated too short. Methods exist for compensating the effects of the troposphere [8], [7].

\section{B. Ground MPC}

Ground multipath propagation originates from a reflection off the ground with (for most practical link distances) a short delay relative to the delay of the LoS signal; the phase of the (received) ground multipath component varies in a different way compared to the phase of the (received) LoS signal while the aircraft moves. The superposition of both signal components results in a fading of the received LoS signal (known as the 2-ray effect). However, measurements have shown that the ground MPC is not always present or exhibits only weak power levels. This may due to a poorly reflecting ground surface (given the current angle of incidence and ground material) or a shadowing of the ground component, e.g. by a building or terrain features.
Proposed Modeling: We propose modeling ground multipath propagation by characterization of reflecting areas on the ground. A ground MPC is received if the ground reflection point lies in such a reflecting area. The ground reflection point is defined as the point where the ground MPC is reflected off the ground surface. The location of the ground reflection point depends on both ground station antenna height above ground and aircraft position. Thus, once the reflecting areas are defined, the interaction of the ground MPC with the LoS component, and the resulting attenuation or amplification of the latter, follows directly from the underlying geometry.

The properties of reflecting areas are given in the following. The actual values for the different properties are drawn from statistical distributions derived from measurement data (see Fig. 2). See Fig. 2 for an example of the reflecting areas. Note that all of those properties strongly depend on the environment a ground station is set up in.

- Coverage ratio: We define the coverage ratio as the ratio between the sum of all reflecting area sizes and the overall area size around the ground station.

- Size: We model the size of a reflecting area statistically.

- Shape: The reflecting areas (such as a runway, a parking area, a taxiing area) are of different shape. We also model the distribution of the shape statistically.

- Material: The ground station environment is usually composed of a mix of different types of surfaces. Their electro-magnetic properties such as conductivity and permitivity have been extensively investigated in the past [9], [10] and are modeled by statistical distributions.

- Roughness: The roughness has an influence on how much power is scattered in all directions rather than reflected in the direction defined by the law of reflection [11]. We describe the roughness as a statistical parameter.

The distributions of the above parameters can be derived from measurement data. Hereby, we first calculate the reflection coefficient of the ground surface causing the ground MPC for a given aircraft position. Depending on the bandwidth of the channel measurements, the reflection coefficient can either be measured directly or estimated based on the received LoS power variation.

If no measurement data exists, the above distributions can also be estimated from a real-world scenario. For example, if it is known that a ground station is located directly on a shore line about half of the surrounding ground surface will be modeled as a water surface with its respective electro-magnetic properties.

Fig. 2 shows one realization of the ground reflecting areas for the environment flight measurements were taken [7]. Additionally, the dashed line in Fig. 2 shows the track of the ground reflection point if an aircraft is flying on a straight track past the ground station (antenna height: $25 \mathrm{~m}$, minimum range: $30 \mathrm{~km}$, aircraft altitude: $5 \mathrm{~km}$ ).

\section{Lateral MPC}

By lateral MPCs we mean signal components that originate from reflections outside the two-dimensional plane defined by 


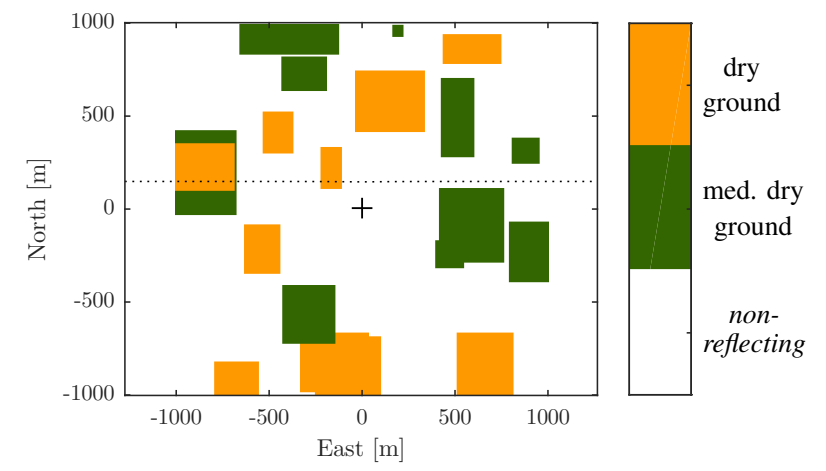

Fig. 2: Visualization of reflecting areas modeling the effect of ground multipath propagation.

the LoS path and ground MPC. In most cases lateral MPCs are attributable to buildings, large structures, vegetation, or terrain; unlike the ground MPC, lateral MPCs have a relatively large delay compared to the LoS signal. Note that lateral MPCs may cause large range estimation errors for terrestrial radionavigation systems, e.g. distance measurement equipment (DME) [3].

Proposed Modeling: It can be observed that lateral MPCs with similar parameters for the delay, Doppler frequency and amplitude, only appear within specific sections of the flight; if the aircraft returns to the approximately same location, MPCs with similar parameters will be observed again. We therefore propose modeling lateral components as point reflectors with a cone shaped opening [12]. A point reflector can be described by the following parameters:

- Location: The location of a point reflector defines the excess delay (relative to the LoS signal) of the generated MPC.

- Opening direction: The opening direction is the direction of the cone's axis of symmetry. The parameter is characterized by an azimuth and elevation angle, as seen from the reflector location.

- Opening angle: The opening angle describes how far the cone opens in terms of azimuth and elevation.

- Power: The power of a reflector is described as a statistical distribution. This distribution includes a temporal correlation. Depending on aircraft and reflector location, the power is additionally altered by FSPL and antenna gains.

The derivation of the distributions of the above parameters requires knowledge about the locations of objects causing lateral MPCs. To calculate those locations from the measurement data we have developed a two-stage algorithm. First, the MPC parameters delay, Doppler frequency, and amplitude are calculated using a super-resolution parameter estimation and tracking algorithm similar to [13]. Based on those parameters we use Bayesian techniques to estimate a probability density function (PDF) expressing the probability of a reflector to be in a certain location in space, as shown in Fig. 3. This figure reveals that in this example the reflector is a hangar building.

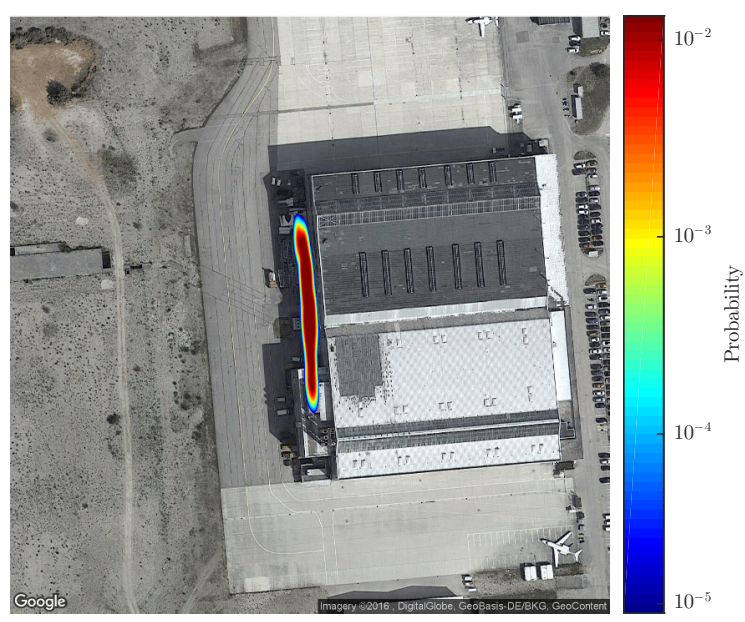

Fig. 3: Estimated distribution of the reflector location.

Given the locations of all reflectors, the ground antenna and the aircraft location as well as the received power levels, the distributions of the above parameters can be derived. Measurements reveal that the vast majority of reflectors is located within about $2 \mathrm{~km}$ of the ground station. The reflector density decreases quickly for an increasing distance between ground station and reflector. Additionally, the data shows that most reflectors open roughly in the direction under which the ground station is seen from the reflector.

\section{Antenna Patterns}

The ground and airborne antennas radiation patterns have a significant influence on the received power level and, thus, are taken into account in the proposed modeling approach ${ }^{1}$. While the pattern of the antenna on the ground for typical link geometries exhibits only small variations for typical link distances, the aircraft antenna pattern is not homogeneous at all: fades exceeding $10 \mathrm{~dB}$ for a duration of more than $20 \mathrm{~s}$ were observed while the aircraft was performing banking maneuvers [7]. In unfavorable circumstances, the aircraft pattern may attenuate the LoS signal, while at the same time amplifying a MPC thus resulting in very challenging propagation conditions for CNS systems.

Due to the geometrical nature of the model, the inclusion of radiation patterns is straight forward. The radiation pattern may only describe received amplitude but can also include information about the phase as well. If the locations of aircraft and reflector relative to the ground station are known, the gain of the ground antenna can be directly calculated for all propagation paths. For the calculation of the airborne pattern gain the aircraft attitude, i.e. yaw, pitch, and roll, is additionally required.

\section{E. Ground Shadowing}

Ground shadowing is understood as the blockage of the LoS path by surrounding structures, vegetation or terrain. Ground

\footnotetext{
${ }^{1}$ The airborne radiation pattern may include also shadowing effects caused by the airframe.
} 


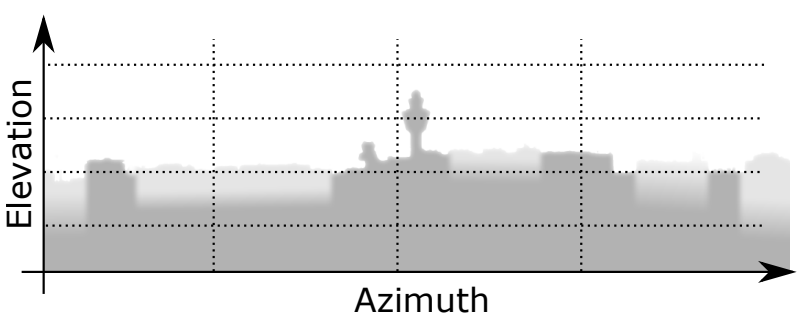

Fig. 4: Azimuth-elevation mask describing the attenuation of the LoS signal.

shadowing usually causes a strong attenuation or even full blockage of the LoS path. The blocking object has to be above the antenna in terms of elevation to be able to obstruct the LoS path.

Ground shadowing has been observed to severely degrade the ranging performance, as it can lead to a strong attenuation of the LoS signal for large areas. This especially applies for aircraft flying at low elevation angles relative to the ground facility, a typical scenario when using terrestrial radionaviation. A lateral MPC may be received with a higher power $P_{\mathrm{M}, \mathrm{Fs}}$ than the LoS power $P_{\mathrm{L}, \mathrm{Fs}}$. An example of this effect has been observed in measurements described in [3]. Herein, the LoS is strongly attenuated by vegetation, while a lateral MPC exists originating from a hangar: the LoS signal is received at a lower power than the lateral MPC for over a minute. The result is a strongly degraded DME performance, i.e. errors exceeding $150 \mathrm{~m}$.

Proposed Modeling: We propose modeling ground shadowing by an azimuth-elevation mask. An example for such a mask is shown in Fig. 4. Similar to an antenna radiation pattern the brightness in Fig. 4 describes the attenuation of the LoS signal. Thus, for any aircraft location the LoS signal attenuation can be directly calculated. The elevation mask can be derived from channel measurements, digital elevation model (DEM) data, or image processing techniques, e.g. deriving it from a panoramic image taken from the ground station location.

\section{F. Colored Noise \& Diffuse MPCs}

Diffuse MPCs are caused by clusters of point reflectors or scattering. Thus, diffuse MPCs can usually not be modeled as a single point reflector. As their power level is generally significantly lower than the power levels of other types of MPCs, their influence on the performance of CNS systems is minor.

Proposed Modeling: We propose modeling diffuse MPCs as a purely statistical process. The colored noise component can be derived by first removing all MPCs from the measurement data. Based on the residual signal we characterize the statistics of the colored noise process and describe the correlation in terms of delay and time.

Fig. 5 shows the filtered scattering function of the colored noise after the specular components are removed from the measurement signal. Both delay and Doppler frequency are

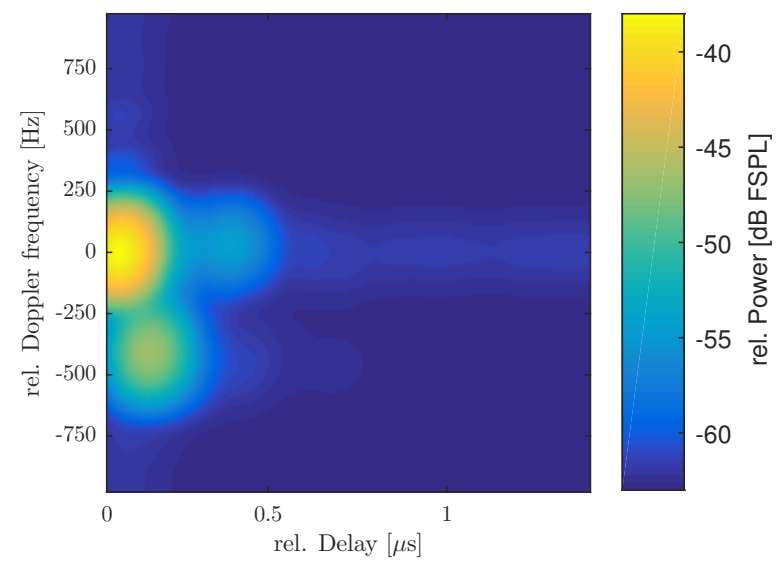

Fig. 5: Scattering function of the filtered colored noise in the residual measurement data.

taken relative to LoS signal parameters. The process is modeled as moving average process, in both delay and frequency direction. From Fig. 5 we can observe three major clusters: the first is centered at a delay of about $0.1 \mu \mathrm{s}$ at a Doppler frequency of $0 \mathrm{~Hz}$ is caused by objects in the direct vicinity of the ground station. The other two clusters are caused by two larger buildings in the ground station environment.

\section{RESULTS}

As the work is still ongoing, we do not provide any distributions of the various channel model parameters. However, in this section we present preliminary results using the modeling approach described in Sec. II.

Fig. 6 shows the delay and Doppler frequency of all MPCs detected in a $25 \mathrm{~s}$ segment of measurement data. Different colors are used to distinguish between different propagation paths. Within that segment the aircraft is performing a flyby of the ground station at close distance and low altitude (compare takeoff \& landing (TL) scenario in [7]). All MPC parameters are taken relative to the LoS signal parameters. Fig. 7 shows a simulation of the propagation conditions for the same segment based on the proposed modeling approach.

The results presented are preliminary in that sense that the distributions of the parameters described in Sec. II are only derived from a relatively short segment of the measurement data of about $60 \mathrm{~s}$. Therefore, Fig. 7 is to be interpreted as a proof-of-concept.

Both Fig. 7 and Fig. 6 look qualitatively very similar even though the reflectors in the channel simulations are distributed randomly. Distributions for their distance to the ground station and opening directions and angles are derived from measurement data.

We observe that the proposed modeling approach is able to capture the time variant nature of the channel. Depending on the geometry, MPCs may be visible for a several seconds. The rapidly changing propagation conditions of the channel are due to the small distance between aircraft and ground station of less than $2 \mathrm{~km}$. 


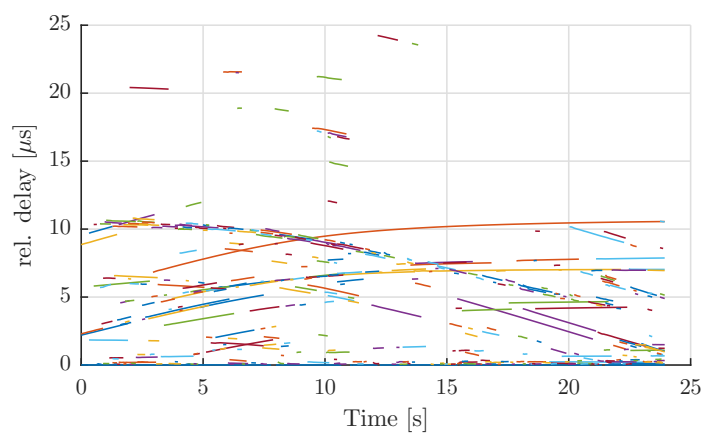

(a) Delay

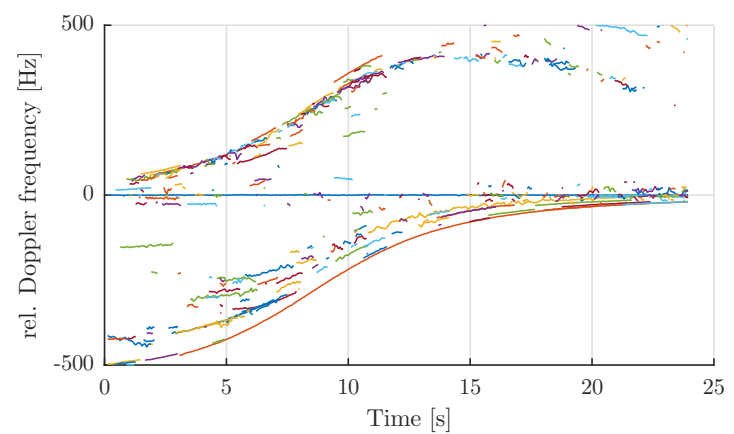

(b) Doppler frequency

Fig. 6: Measured propagation paths and their corresponding parameters based on a super-resolution parameter estimation.

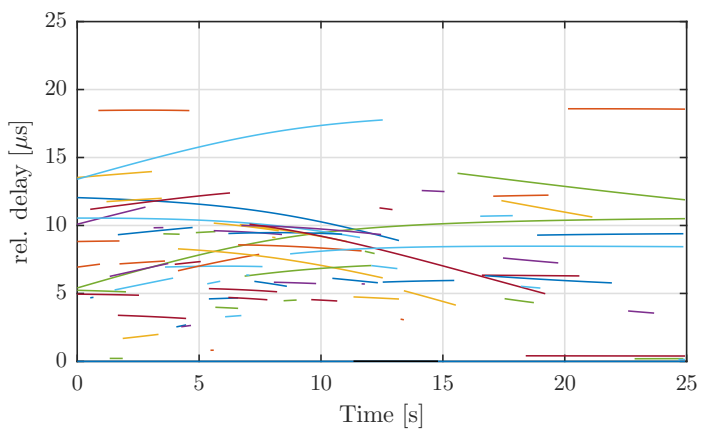

(a) Delay

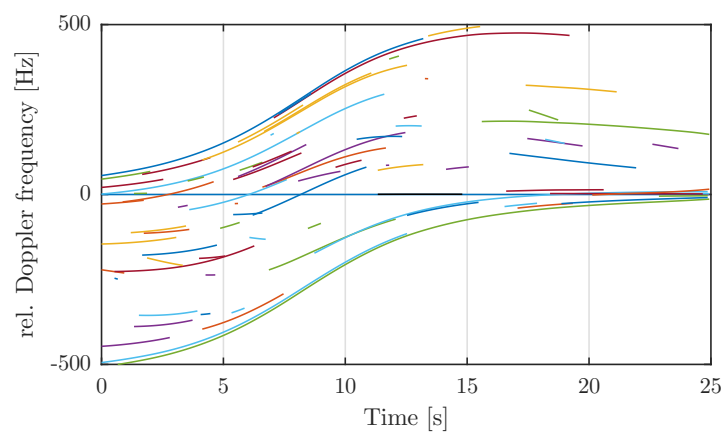

(b) Doppler frequency

Fig. 7: Propagation paths and their corresponding parameters based on simulations using the proposed channel modeling approach. The ground MPC is marked in black.

The ground MPC in Fig. 7 between $10 \mathrm{~s}$ and $15 \mathrm{~s}$ is plotted in black. However, as the ground MPC has almost the identical delay and Doppler frequency as the LoS signal, the ground MPC cannot be separated from the LoS signal in the measurement data in Fig. 6. In measurements the ground MPC manifests itself by a variation of the received LoS power (not shown).

\section{CONCLUSION AND OUTLOOK}

In this contribution we describe a geometrical-statistical approach of modeling the AG channel. The model is to be used to analyze the performance of CNS systems in aviation. We propose how the parameters can be derived from measurement data. Preliminary results demonstrate how well the geometrical-statistical modeling approach is able to capture the time variant nature of the channel.

Future work includes analyzing the full duration of measurement data by applying the methods described in Sec. II. Based on the results the final distributions of the model parameters can be derived.

\section{REFERENCES}

[1] M. Schnell, U. Epple, D. Shutin, and N. Schneckenburger, "LDACS: Future Aeronautical Communications for Air-Traffic Management," IEEE Commun. Mag., vol. 52, no. 5, pp. 104-110, 2014.
[2] S. Lo, Y.-H. Chen, P. Enge, B. Peterson, and R. Erikson, "Distance Measuring Equipment Accuracy Performance Today and for Future Alternative Position Navigation and Timing (APNT)," in ION GNSS+, (Nashville (TN), USA), 2013.

[3] W. Pelgrum, K. Li, M. Smearcheck, and F. van Graas, "eDME architecture development and flight-test evaluation," in ION GNSS+, (Nashville (TN), USA), 2012.

[4] J. Proakis, Digital Communications. Boston, MA: McGraw-Hill, 4th ed. 2010.

[5] P. Misra and P. Enge, Global Positioning System: Signals, Measurements, and Performance. Lincoln (MA), USA: Ganga-Jamuna Press, 2nd ed., 2011.

[6] D. W. Matolak and R. Sun, "Air-Ground Channel Characterization for Unmanned Aircraft SystemsPart I: Methods, Measurements, and Models for Over-water Settings," IEEE Trans. Veh. Technol. (accepted for publication), vol. PP, no. 99, 2016

[7] N. Schneckenburger, T. Jost, D. Shutin, M. Walter, T. Thiasiriphet, M. Schnell, and U.-C. Fiebig, "Measurement of the L-band air-to-ground channel for positioning applications," IEEE Trans. Aerosp. Electron. Syst. (accepted), 2016.

[8] G. H. Millman, "Atmospheric effects on VHF and UHF propagation," in IEEERE International Convention, vol. 46, pp. 1492-1501, 1958.

[9] ITU-R, "Report P.1008-1: Reflection from the surface of the Earth," tech. rep., 1990.

[10] ITU-R, "Recommendation P.527-3: Electrical characteristics of the surface of the earth," tech. rep., 1992.

[11] J. D. Parsons, The Mobile Radio Propagation Channel. Wiley, 2nd ed. 2000.

[12] T. Jost, W. Wang, U.-C. Fiebig, and F. Pérez-Fontán, "A Wideband Satellite-to-Indoor Channel Model for Navigation Applications," IEEE Trans. Antennas Propag., vol. 62, no. 10, pp. 5307-5320, 2014.

[13] T. Jost, W. Wang, U.-C. Fiebig, and F. Pérez-Fontán, "Detection and tracking of mobile propagation channel paths," IEEE Trans. Antennas Propag., vol. 60, no. 10, pp. 4875-4883, 2012. 